\title{
Universal optical setup for phase-shifting and spatial-carrier digital speckle pattern interferometry
}

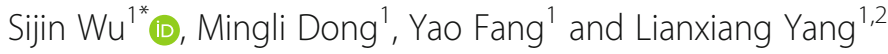

\begin{abstract}
Background: Digital speckle pattern interferometry (DSPI) is a competitive optical tool for full-field deformation measurement. The two main types of DSPI, phase-shifting DSPI (PS-DSPI) and spatial-carrier DSPI (SC-DSPI), are distinguished by their unique optical setups and methods of phase determination. Each DSPI type has its limited ability in practical applications.

Results: We designed a universal optical setup that is suitable for both PS-DSPI and SC-DSPI, with the aim of integrating their respective advantages, including PS-DSPI's precise measurement and SC-DSPI's synchronous measurement, improving DSPI's measuring capacity in engineering.
\end{abstract}

Conclusion: The proposed setup also has several other advantages, including a simple and robust structure, easy adjustment and operation, and versatility of measuring approach.

Keywords: Digital speckle pattern interferometry, Phase shift, Spatial carrier, Deformation measurement, Universal optical setup, Digital holographic interferometry

\section{Background}

Deformation measurement, especially three-dimensional (3D) deformation measurement, is essential to the quantitative description of object change and the accurate determination of mechanical properties. Traditionally, deformation measurement is carried out by the use of displacement transducers, such as strain gauges [1]. However, displacement transducers suffer from the disadvantage of being a spot measurement technique, which leads to low spatial resolution and insufficient information for full-field deformation measurement. Optical techniques such as digital speckle pattern interferometry (DSPI) [2, 3], digital image correlation [4], and Moiré method [5] have become preponderant methods in the measurement of deformation for objects with rough surfaces due to their full-field, stand-off, and non-contact measurement nature. Moreover, optical methods, particularly DSPI, are also very precise tools. DSPI is mainly divided, based on their optical setups and interferometric phase extraction methods, into

\footnotetext{
* Correspondence: swu@bistu.edu.cn

${ }^{1}$ School of Instrumentation Science and Opto-electronics Engineering, Beijing Information Science and Technology University, Beijing 100192, China Full list of author information is available at the end of the article
}

two categories: phase-shifting DSPI (PS-DSPI) and spatialcarrier DSPI (SC-DSPI). The SC-DSPI is also known as digital holographic interferometry $[6,7]$.

PS-DSPI utilizes the interference between an object beam from a measuring target and a reference beam from a fixed surface to measure the out-of-plane deformation, and the interference between object and reference beams from the measuring target via different paths to measure in-plane deformations $[8,9]$. 3D deformation measurement is then realized by combining one optical setup for out-of-plane deformation measurement and two optical setups for in-plane deformation measurement together. The three channels are enabled in turn when performing the 3D measurement, resulting in asynchronous measurement of the 3D deformations. However, synchronous measurement of 3D deformations is desired in practical applications to enable the change and mechanical model of the measuring object to be characterized properly. Therefore, the inability of PSDSPI to perform synchronous measurement limits its employment in practical engineering. Furthermore, PSDSPI is usually unsuitable for dynamic measurement due to the amount of time consumed in the process of 
obtaining the interferometric phase. The dominant phase extraction method in PS-DSPI is the temporal phase shift, which carries out several phase shifts and requires the measuring target to be stationary during the phase shift [10]. Dynamic deformations are not easily measured, even if the time interval between adjacent phase steps is very short. Though other phase extraction methods, such as spatial phase shift [11] and phase of difference phase shift [12], have been used in DSPI to make dynamic deformation measurement possible, these methods are difficult to use, result in a more complicated system structure, and provide less reliable measurement results. Consequently, these fast phase extraction methods are rarely used in commercial PS-DSPI instruments.

SC-DSPI also uses a multi-channel optical setup, usually a three-channel setup, to measure 3D deformations $[13,14]$. The three channels work simultaneously, and three speckle interferograms are recorded in an image frame. The information of the three interferograms can be separated in the frequency domain, and their corresponding phase maps can later be calculated if proper spatial carrier frequencies are used [15]. The combination of the three phase maps allows the final 3D deformation to be obtained. SC-DSPI's measurement characteristics make synchronous measurement of 3D deformations possible because the three speckle interferograms are recorded together in one frame and the three phase maps are obtained simultaneously. Dynamic measurement of deformations is also possible because only one image frame is used to measure deformations, eliminating the need for a specified time interval [16]. The dynamic measurement speed depends on the camera frame rate. Though SC-DSPI outperforms PS-DSPI in terms of synchronous and dynamic measurement, its disadvantages include a lower-quality phase map [17], greater loss of laser energy, and much smaller measuring area, thus limiting its use in practical applications.

PS-DSPI and SC-DSPI have their respective characteristics and are employed in different applications. However, their respective defects limit their wide use in engineering. Their area of application could be expanded if both techniques could be combined together. However, this idea is not easy to realize due to their distinct optical setup.

We have built a universal optical setup for both PSDSPI and SC-DSPI. 3D deformations can be measured by this optical setup using either PS-DSPI or SC-DSPI. Thus the flexibility of deformation measurement in engineering is fulfilled by the use of the proposed optical setup. The optical setup is also very simple, robust, and easy to use.

\section{Method}

\section{Arrangement of universal optical setup}

The universal optical setup for PS-DSPI and SC-DSPI adopts a three-channel optical arrangement. Each channel consists of an object and reference beam pair derived from an individual laser. Components in each channel are almost the same, but the laser wavelength can be different. The incident angles, or illumination angles, of the three object beams striking the measuring target are artificially arranged to achieve optimal 3D deformation measurement results. The illumination angles will be discussed later.

The optical arrangement of the universal optical setup is depicted in Fig. 1. Considering the similarity of the three channels, the optical arrangement of only one

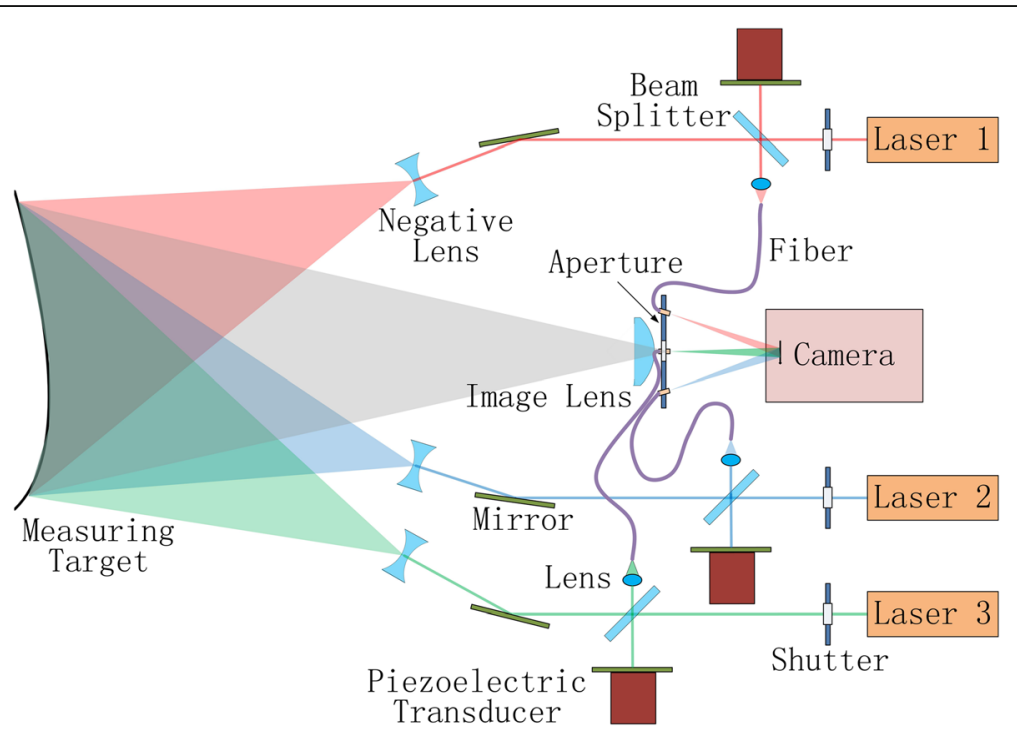

Fig. 1 Universal optical setup for phase-shifting and spatial-carrier digital speckle pattern interferometry 
channel is described to show the optical interference process. The laser beam is divided into object and reference beams by a beam splitter. The object beam then strikes the measuring target after being expanded by a negative lens or other optical components or parts with similar function, such as a microscope objective. The scattered light from the target is collected by an imaging lens, such as an aspheric lens, then reaches the image sensor of the camera via an aperture. The aperture works as a regulator of light intensity in PS-DSPI mode and a filter of spatial frequency in SC-DSPI mode. The reference beam is coupled into an optical fiber via a piezoelectric-transducer-driven mirror. The elongation of the piezoelectric transducer (PZT) is automatically controlled by a computer to modulate the optical path of the reference beam, resulting in the phase shift in the PS-DSPI measurement. The emergent light from the fiber strikes the camera sensor at a small angle between it and the optical axis. This angle determines the carrier frequency, a key parameter in the SC-DSPI measurement. The object and reference beams encounter each other on the camera sensor, resulting in optical interference. The generated speckle interferograms are captured by the camera and recorded by the computer for further processing.

The other two channels follow the same principle, but have different illumination angles and reference beam incident angles. The differences in the incident angles of the reference beams guarantee the separation of the interferometric signals from the three channels in the frequency domain, when the setup works in the SCDSPI mode. The illumination angle differences among the three channels result in different displacement sensitivity coefficients. The combination of these displacement sensitivity coefficients forms a displacement sensitivity matrix with which the relationship between the 3D deformations and the interferometric phases obtained by PS-DSPI and SC-DSPI is built. The phase determination and deformation calculation procedures are discussed in the next section. Various illumination angle combinations among the three channels yield different displacement sensitivity matrices. Among these combinations, right-angle distribution and homogeneous distribution, described in Fig. 2, are the two simplest and optimal arrangements. In both types, the magnitudes of the illumination angles are equal, but the directions differ.

When PS-DSPI is used to measure 3D deformations, the three channels are enabled in turn by opening the shutters in front of each laser. Only one interferogram, generated by a pair of object and reference beams from a channel, is captured by the camera at a time. The implementation of a round of measurements using the three channels in turn yields three equations which express the mathematical relationship between the interferometric phases and image intensities. When SC-DSPI

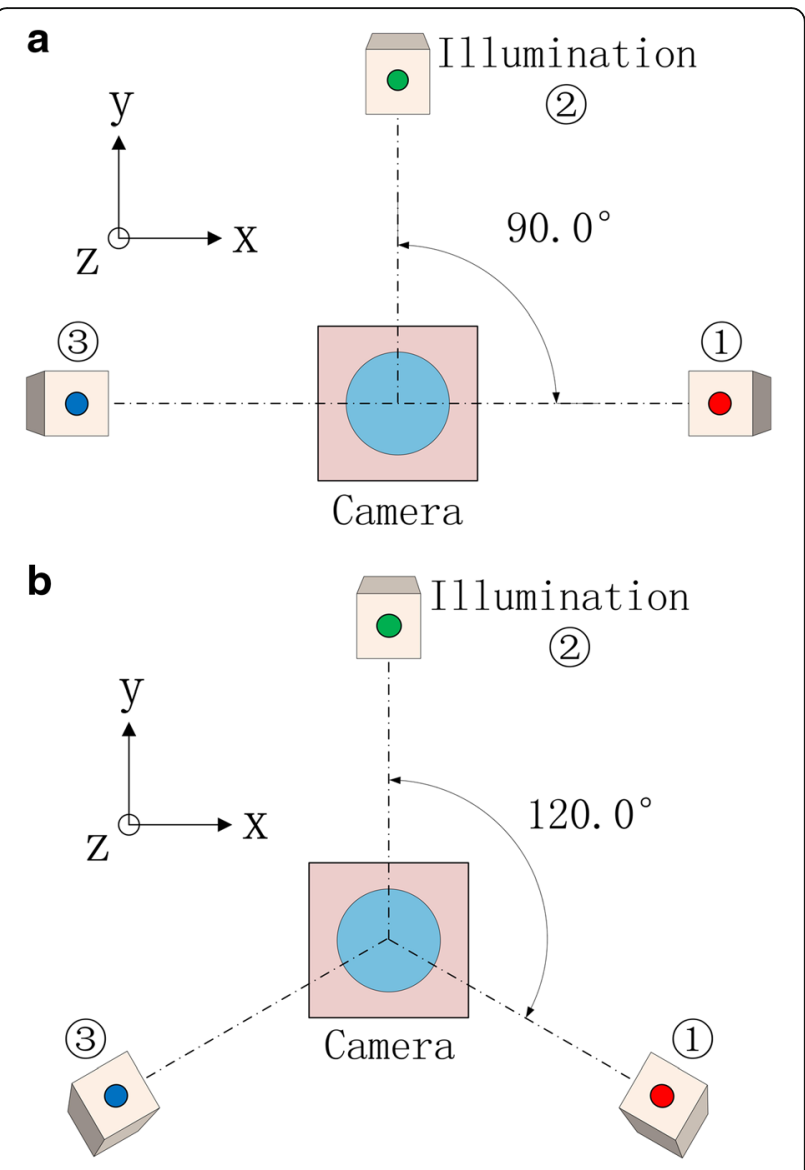

Fig. 2 Typical illumination layouts for the universal optical setup. a Right angle distribution. $\mathbf{b}$ Homogeneous distribution

is used for measurement, the three shutters are opened together, resulting in three pairs of object and reference beams emerging on the camera sensor simultaneously. Each object beam- reference beam pair generates an interferogram, resulting in the simultaneous recording of three independent interferograms. The three interferograms are later separated in the frequency domain after a Fourier transform is performed on them. The interferometric phases are extracted from the separated interferograms after an inverse Fourier transform is performed.

\section{Phase determination using PS-DSPI}

The interferogram generated by the PS-DSPI can be expressed as

$$
\begin{aligned}
I(x, y)= & I_{0}(x, y) \\
& +B(x, y) \cos \left[\phi(x, y)+2 \pi f_{x} x+2 \pi f_{y} y\right],
\end{aligned}
$$

where $I(x, y)$ is the intensity distribution of the interferogram, $I_{0}(x, y)$ is the background light, $B(x, y)$ is a coefficient correlating with the contrast, $\phi(x, y)$ is the 
interferometric phase, $f_{x}$ and $f_{y}$ indicate the carrier frequencies which are introduced by the slightly deflected reference beams, and $(x, y)$ indicates the two-dimensional distribution.

The interferogram intensity $I(x, y)$ is recorded by the camera, and the carrier frequencies $f_{x}$ and $f_{y}$ are determined by the incidence angle of the reference beam, but the three remaining variables in Eq. (1) are unknown, making the equation unsolvable. Additional conditions need to be added to resolve this problem. Typically, the additional condition is a series of artificial phase changes. The method to solve the equation by artificially changing the interferometric phase is known as phase shifting. This method can be further divided into temporal and spatial phase shifting. The temporal phase shifting, which changes the phase over time, is the dominant phase determination method in PS-DSPI due to its ease of use and ability to formulate high-quality phase maps. The number of steps and phase change intervals are multifarious [18]. For example, the popular four-step temporal phase shift changes the phase four times with an interval of $\pi / 2$. As a result, four equations are obtained as

$$
\begin{aligned}
I_{i}(x, y)= & I_{0}(x, y) \\
& +B(x, y) \cos \left[\phi(x, y)+2 \pi f_{x} x+2 \pi f_{y} y+(i-1) \frac{\pi}{2}\right],(i=1,2,3,4) .
\end{aligned}
$$

Solving Eq. (2) for $\phi(x, y)$ results in the following expression:

$$
\phi(x, y)=\tan ^{-1} \frac{I_{4}(x, y)-I_{2}(x, y)}{I_{1}(x, y)-I_{3}(x, y)} .
$$

After the measuring target has been deformed, the phase shift is carried out again to determine the interferometric phase according to the deformed state. The phase difference is then determined by simply subtracting the phase before deformation from the phase after deformation. This is expressed as

$$
\Delta \phi_{1}(x, y)=\phi_{a}(x, y)-\phi_{b}(x, y)
$$

where $\phi_{a}(x, y)$ and $\phi_{b}(x, y)$ are the phase distributions after and before the deformation, respectively. The other two phase differences $\Delta \phi_{2}(x, y)$ and $\Delta \phi_{3}(x, y)$ are determined by performing the same procedure on the other channels.

In the proposed universal optical setup, the phase shift is carried out by the PZT. A PZT elongation of $\lambda / 8$, where $\lambda$ is the laser wavelength, causes a phase shift of $\pi / 2$, which is the amount required by the four-step temporal phase shift. Fine control of a well-calibrated PZT aids in the precise determination of the interferometric phase using PS-DSPI.

\section{Phase determination using SC-DSPI}

Due to the simultaneous recording of the three interferograms in the SC-DSPI mode, the image intensity is the sum of all interferograms, which is expressed by

$$
\begin{aligned}
I_{s}(x, y)= & I_{s 0}(x, y) \\
& +\sum_{i=1}^{3} B_{i}(x, y) \cos \left[\phi_{i}(x, y)+2 \pi f_{i x} x+2 \pi f_{i y} y\right],
\end{aligned}
$$

where $I_{s 0}(x, y)$ is the sum of the background lights.

Aided by Euler's formula, Eq. (5) can be transformed to

$$
\begin{aligned}
I_{s}(x, y)= & I_{s 0}(x, y) \\
& +\sum_{i=1}^{3}\left[C_{i}(x, y) e^{j 2 \pi\left(f_{i x} x+f_{i y} y\right)}+C_{i}^{*}(x, y) e^{-j 2 \pi\left(f_{i x} x+f_{i y} y\right)}\right],
\end{aligned}
$$

where $C_{i}(x, y)=B_{i}(x, y) \exp [j \phi(x, y)] / 2$, " denotes the complex conjugate.

After a Fourier transform is performed, Eq. (6) is transformed to

$$
\begin{aligned}
F\left(f_{\xi}, f_{\eta}\right)= & F T\left[I_{s}(x, y)\right]=\mathrm{A}\left(f_{\xi}, f_{\eta}\right) \\
& +\sum_{i=1}^{3}\left[P_{i}\left(f_{\xi}-f_{i x}, f_{\eta}-f_{i y}\right),\right. \\
& \left.+Q_{i}\left(f_{\xi}+f_{i x}, f_{\eta}+f_{i y}\right)\right]
\end{aligned}
$$

where $F T$ denotes the operation of Fourier transform, $\left(f_{\xi}, f_{\eta}\right)$ are the coordinates in the frequency domain, and

$$
\left\{\begin{array}{rl}
\mathrm{A}\left(f_{\xi}, f_{\eta}\right) & =F T\left[I_{s 0}(x, y)\right] \\
P_{i}\left(f_{\xi}-f_{i x}, f_{\eta}-f_{i y}\right) & =F T\left[C_{i}(x, y) e^{j 2 \pi\left(f_{i x} x+f_{i y} y\right)}\right] \\
Q_{i}\left(f_{\xi}+f_{i x}, f_{\eta}+f_{i y}\right) & =F T\left[C_{i}^{*}(x, y) e^{-j 2 \pi\left(f_{i x} x+f_{i y} y\right)}\right]
\end{array},\right.
$$

Eq. (7) shows there are a total of seven components in the frequency domain, where $P_{i}\left(f_{\xi}-f_{i x}, f_{\eta}-f_{i y}\right)$ and $Q_{i}\left(f_{\xi}+\right.$ $\left.f_{i x}, f_{\eta}+f_{i y}\right)$ are three pairs of conjugate components and $\mathrm{A}\left(f_{x}, f_{y}\right)$ represents the low-frequency background signal. The locations of $P_{i}\left(f_{\xi}-f_{i x}, f_{\eta}-f_{i y}\right)$ and $Q_{i}\left(f_{\xi}+f_{i x}, f_{\eta}+f_{i y}\right)$ are determined by the carrier frequencies $f_{i x}$ and $f_{i y}$. All seven components can be well separated by fine adjustment of the incidence angles of the reference beams and the aperture in the universal optical setup. To intuitively describe the frequency spectrum obtained by SC-DSPI, Fig. 3 illustrates a distribution of the seven components that was generated by the proposed optical setup in an experiment. More information about the synchronous recording and separation of the multiple interferograms can be found in Refs. [19] and [20]. 


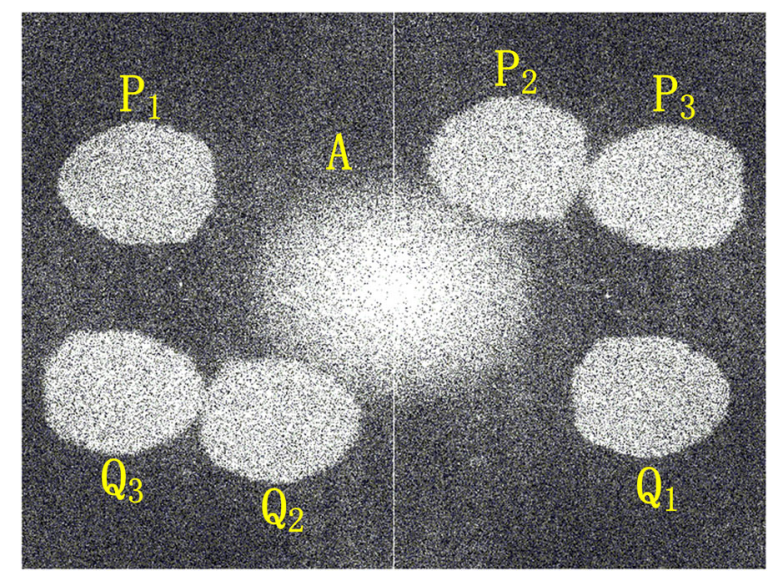

Fig. 3 Frequency spectrum obtained by the SC-DSPI

Since both $P_{i}\left(f_{\xi}-f_{i x}, f_{\eta}-f_{i y}\right)$ and $Q_{i}\left(f_{\xi}+f_{i x}, f_{\eta}+f_{i y}\right)$ contain the same interferometric phase, either of them can be used for phase extraction. This is realized by applying an inverse Fourier transform on the selected component and performing further calculations. For example, if $P_{i}\left(f_{\xi}-f_{i x}, f_{\eta}-f_{i y}\right)$ is chosen, the phase distribution according to the first channel is

$$
\phi_{1}(x, y)=\tan ^{-1} \frac{I M\left[p_{1}(x, y)\right]}{R E\left[p_{1}(x, y)\right]},
$$

where $I M$ and $R E$ denote imaginary and real parts of the complex number and

$$
p_{1}(x, y)=\mathrm{FT}^{-1}\left[P_{1}\left(f_{\xi}-f_{1 x}, f_{\eta}-f_{1 y}\right)\right]
$$

where $\mathrm{FT}^{-1}$ is the inverse Fourier transform operation.

The phases according to the other two channels, as well as the phases after deformation, are obtained by the same means. Finally, three individual phase difference distributions $\Delta \phi_{1}(x, y), \Delta \phi_{2}(x, y)$ and $\Delta \phi_{3}(x, y)$ are determined by subtracting the phases before deformation from the corresponding phases after deformation.
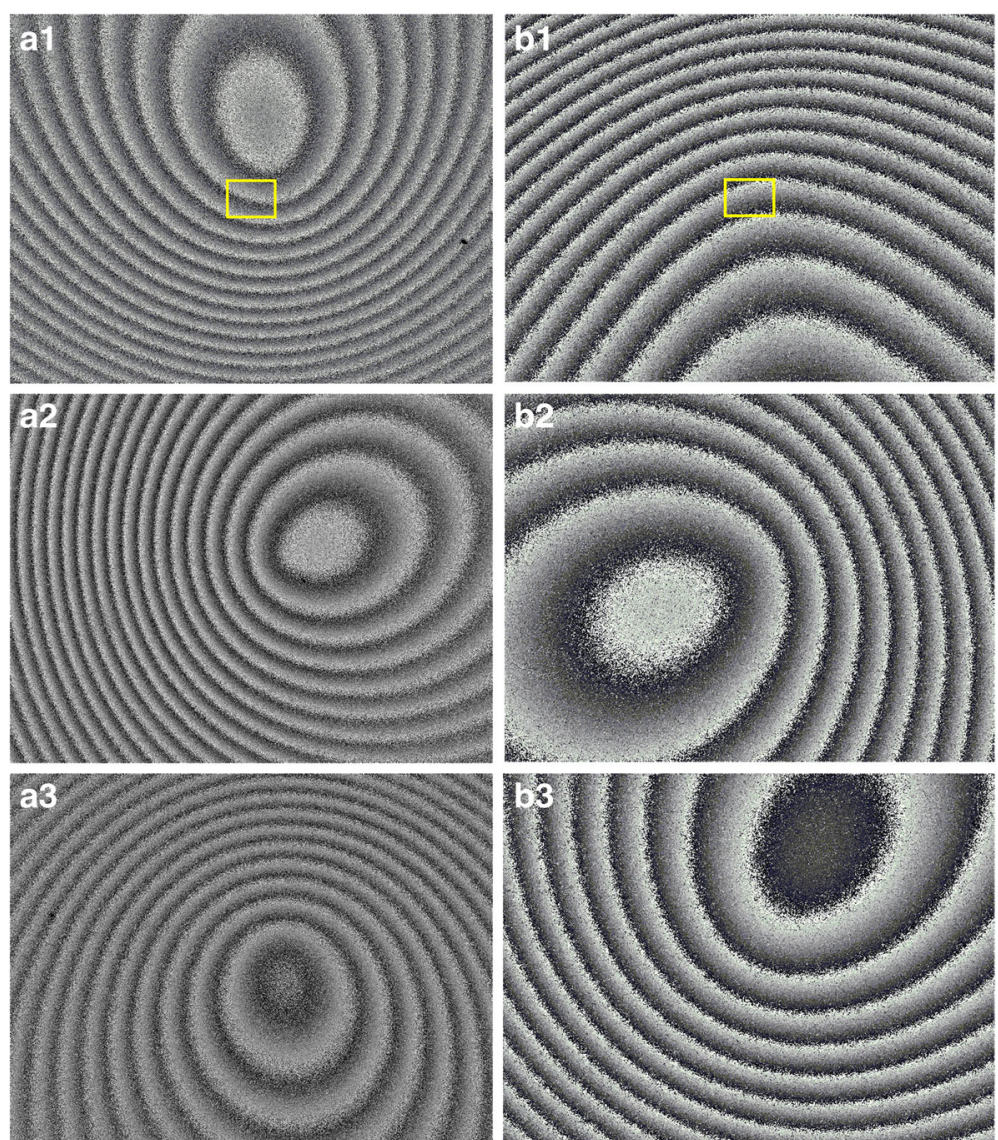

Fig. 4 Original wrapped phase maps. a1 Phase 1 from PS-DSPI. a2 Phase 2 from PS-DSPI. a3 Phase 3 from PS-DSPI. b1 Phase 1 from SC-DSPI. b2 Phase 2 from SC-DSPI. b3 Phase 3 from SC-DSPI 


\section{Calculation of 3D deformations}

The relationship between the deformation and interferometric phase difference in PS-DSPI and SC-DSPI can be expressed by

$$
\Delta \phi(x, y)=\frac{2 \pi}{\lambda} \vec{d}(x, y) \vec{s}(x, y),
$$

where $\Delta \phi(x, y)$ is the phase difference, $\vec{d}(x, y)$ is the deformation vector, and $\vec{s}(x, y)$ is the displacement sensitivity vector, which is dependent on the illumination angles.

If the right-angle-distribution optical arrangement is used, Eq. (11) can be transformed to

$$
\left\{\begin{array}{l}
\Delta \phi_{1}(x, y)=\frac{2 \pi}{\lambda_{1}}[u(x, y) \sin \alpha+w(x, y)(1+\cos \alpha)] \\
\Delta \phi_{2}(x, y)=\frac{2 \pi}{\lambda_{2}}[v(x, y) \sin \alpha+w(x, y)(1+\cos \alpha)], \\
\Delta \phi_{3}(x, y)=\frac{2 \pi}{\lambda_{3}}[u(x, y) \sin (-\alpha)+w(x, y)(1+\cos \alpha)]
\end{array}\right.
$$

where $\lambda_{1}, \lambda_{2}$, and $\lambda_{3}$ are the wavelengths of the three lasers; $u(x, y), v(x, y)$, and $w(x, y)$ are the three components of $\vec{d}(x, y)$ in three dimensions, and $\alpha$ is the illumination angle.

To simplify the calculation, all laser wavelengths are assumed to be the same. This assumption, used with Eq. (12), results in the following expressions for the three deformation vector components:

$$
\left\{\begin{array}{c}
u(x, y)=\frac{\lambda}{4 \pi \sin \alpha}\left[\Delta \phi_{1}(x, y)-\Delta \phi_{3}(x, y)\right] \\
v(x, y)=\frac{\lambda}{4 \pi \sin \alpha}\left[2 \Delta \phi_{2}(x, y)-\Delta \phi_{1}(x, y)-\Delta \phi_{3}(x, y)\right] . \\
w(x, y)=\frac{\lambda}{4 \pi(1+\cos \alpha)}\left[\Delta \phi_{1}(x, y)+\Delta \phi_{3}(x, y)\right]
\end{array}\right.
$$

For the homogeneous-distribution optical arrangement, Eq. (11) becomes

$$
\left\{\begin{aligned}
\Delta \phi_{1}(x, y)= & \frac{2 \pi}{\lambda_{1}}\left[u(x, y) \sin \alpha \cos 30^{\circ}+v(x, y) \sin \alpha \cos 60^{\circ}\right. \\
& +w(x, y)(1+\cos \alpha)] \\
\Delta \phi_{2}(x, y)= & \frac{2 \pi}{\lambda_{2}}[v(x, y) \sin \alpha+w(x, y)(1+\cos \alpha)] \\
\Delta \phi_{3}(x, y)= & \frac{2 \pi}{\lambda_{3}}\left[u(x, y) \sin (-\alpha) \cos 30^{\circ}\right. \\
& \left.+v(x, y) \sin (-\alpha) \cos 60^{\circ}+w(x, y)(1+\cos \alpha)\right] .
\end{aligned}\right.
$$

If the laser wavelengths are assumed to be the same, the deformation vector components have the following expression:

$$
\left\{\begin{array}{l}
u(x, y)=\frac{\sqrt{3} \lambda}{12 \pi \sin \alpha}\left[3 \Delta \phi_{1}(x, y)-2 \Delta \phi_{2}(x, y)-\Delta \phi_{3}(x, y)\right] \\
v(x, y)=\frac{\lambda}{4 \pi \sin \alpha}\left[2 \Delta \phi_{2}(x, y)-\Delta \phi_{1}(x, y)-\Delta \phi_{3}(x, y)\right] \\
w(x, y)=\frac{\lambda}{4 \pi(1+\cos \alpha)}\left[\Delta \phi_{1}(x, y)+\Delta \phi_{3}(x, y)\right]
\end{array} .\right.
$$

The solutions of $v(x, y)$ and $w(x, y)$ are the same for both the right-angle-distribution and homogeneousdistribution types, but the solutions of $u(x, y)$ are different.

\section{Results and Discussion}

An experimental setup based on Fig. 1 and Fig. 2a was built to verify the validity of the presented universal optical setup. Three single-longitudinal-mode diodepumped-solid-state lasers, all with a wavelength of $532 \mathrm{~nm}$, were used as the light sources. A complementary-

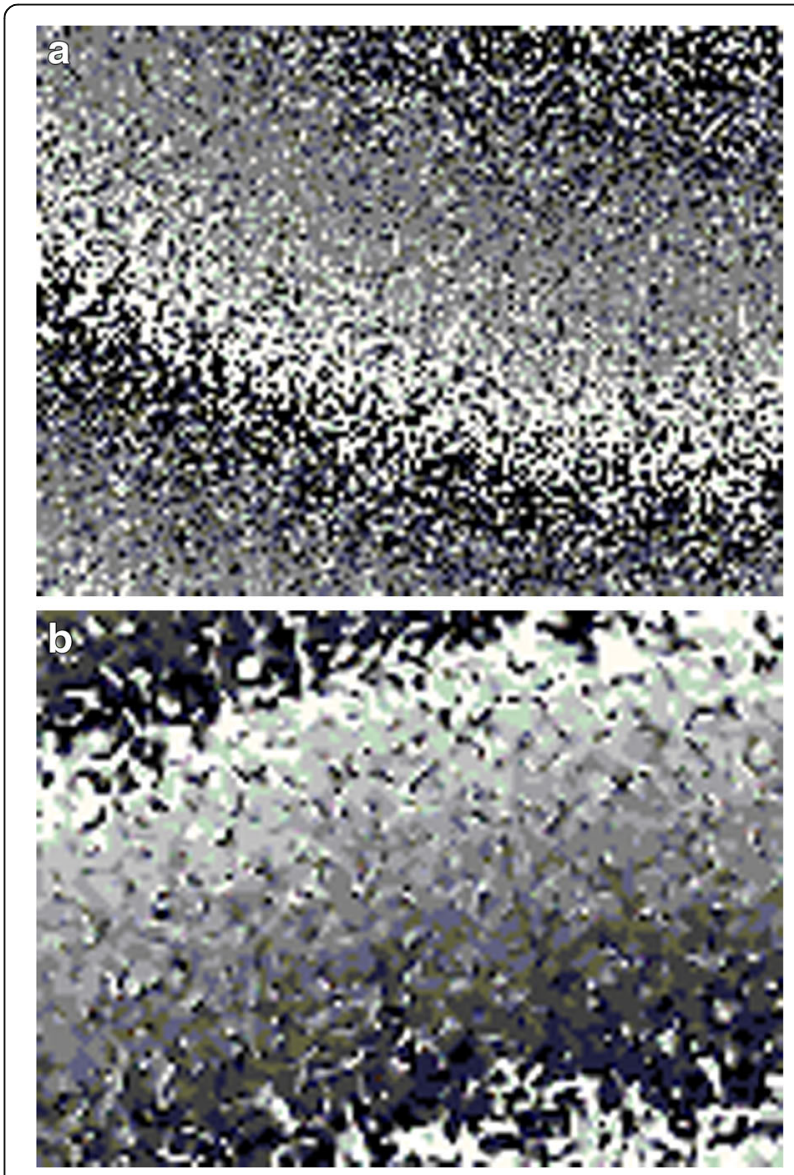

Fig. 5 Comparison of phase maps. a Partial enlargement of phase map from PS-DSPI. $\mathbf{b}$ Partial enlargement of phase map from SC-DSPI 
metal-oxide-semiconductor (CMOS) camera (CatchBEST Co. Ltd., MU3C500M-MRYYO, 500 Mega pixels, $14 \mathrm{fps}$ ) and an aspheric lens with a focus length of $100 \mathrm{~mm}$ were used to capture images. The location of the aspheric lens was carefully adjusted to obtain clear images. Three PZT chips (Thorlabs, Inc., PA4FE, $150 \mathrm{~V}, 2.5 \mu \mathrm{m}$ travel) were used to actuate the phase shifts. The illumination angles were set to around $30^{\circ}$, and the incidence angles of the reference beams were carefully adjusted to guarantee that all components in the frequency domain were well separated. An object with a circular planar surface was used as the measuring target. Out-of-plane deformation $w(x, y)$ was generated by applying a load to the center of the target back, while the in-plane deformations $u(x, y)$ and $v(x, y)$ were generated through rotation of the object surface. All motions were finely controlled using manual micrometer heads. The measuring area in the experiment was $60 \mathrm{~mm} \times 40 \mathrm{~mm}$.

With self-developed programs, both the PS-DSPI and SC-DSPI modes were activated to measure the 3D deformations. The obtained phase differences corresponding to each channel are shown in Fig. 4. Figure 4(a1), (a2) and (a3) are the three phase differences obtained by PSDSPI and Fig. 4(b1), (b2) and (b3) are the phase differences obtained by SC-DSPI. These phase differences are wrapped due to the arc tangent operation expressed in Eqs. (3) and (9). The real phase differences are finally obtained after image smoothing and phase unwrap operations are performed [21]. All of the phase maps in Fig. 4 present clear and regular patterns, illustrating the capability of both PS-DSPI and SC-DSPI to obtain high-quality phase maps. However, differences in image quality between the phase maps obtained by PSDSPI and SC-DSPI can be found after partial enlargement of the original phase maps is processed. Local regions of the phase maps of the same size, corresponding to the first channel in the PS-DSPI and SC-DSPI modes respectively, are marked by yellow boxes in Fig. 4(a1) and (b1). The enlarged parts corresponding to the marked regions, as depicted in Fig. 5, clearly show that the speckle particles in the phase map obtained by PS-DSPI are much smaller than those in the phase map obtained by SCDSPI. This means that the noise in the PS-DSPI phase maps can be filtered more easily than the SC-DSPI's phase maps, or, in other words, the phase smoothing process is performed more times in the SC-DSPI mode, leading to larger error being induced in the phase smoothing process. Consequently, PS-DSPI measurement is usually a1

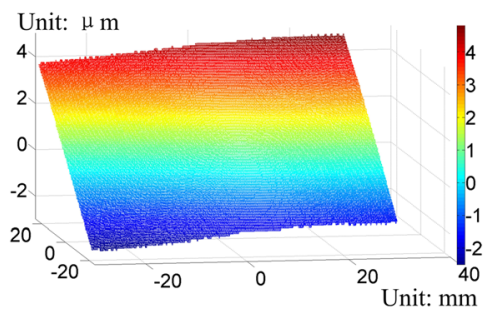

a2

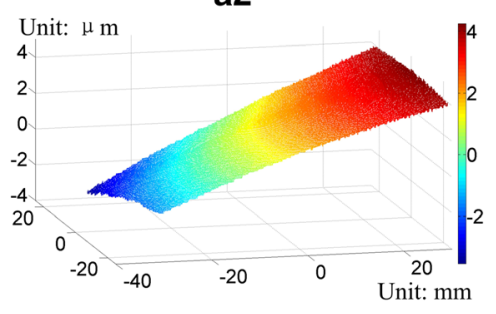

a3

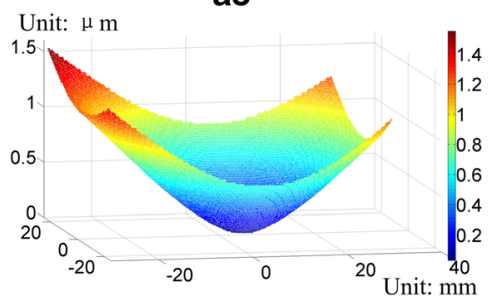

b1

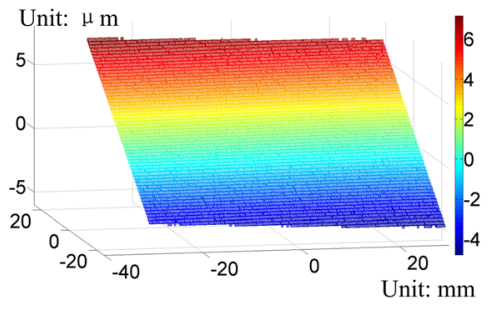

b2

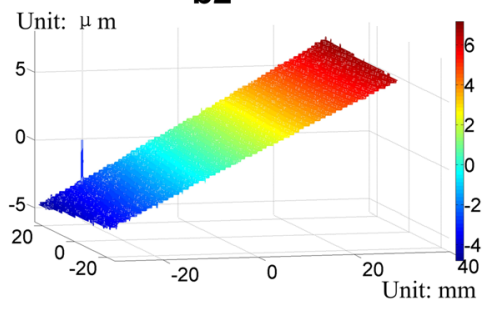

b3

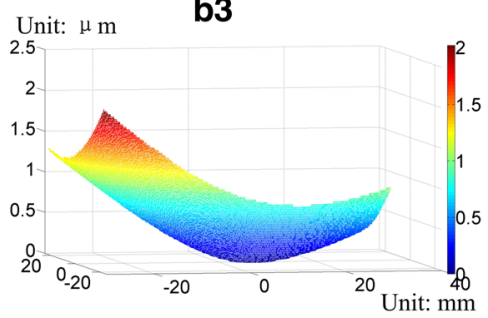

Fig. 6 Height maps for 3D deformations. a1 Deformation $u$ obtained by PS-DSPI. a2 Deformation $v$ obtained by PS-DSPI. a3 Deformation $w$ obtained by PS-DSPI. b1 Deformation $u$ obtained by SC-DSPI. b2 Deformation $v$ obtained by SC-DSPI. b3 Deformation $w$ obtained by SC-DSPI 
more accurate than SC-DSPI measurement. However, SCDSPI reflects its value with its ability to perform dynamic and synchronous 3D deformation measurement.

The final 3D deformations shown in Fig. 6 were determined after the calculations described by Eq. (13) were performed. The horizontal coordinates represent the object surface plane and the vertical coordinates represent the deformation change. Figure 6(a1), (a2) and (a3) show the 3D deformations $u, v$ and $w$ obtained using PS-DSPI, respectively, while Fig. 6(b1), (b2) and (b3) show the 3D deformations obtained using SC-DSPI. The in-plane deformations $u$ and $v$ are orthogonal and vary linearly along the horizontal direction. These results indicate that the magnitude of the relative displacement of the circular surface caused by the rotation increases gradually and linearly. This is in accord with the results of theoretical analysis. The out-of-plane deformation $w$ presents a distribution that decreases gradually from the periphery to the loading center. Though slight differences can be found between the results obtained by PS-DSPI and SC-DSPI due to the impossibility in duplicating the loading, this deformation data proves that reasonable results can be obtained by both PS-DSPI and SC-DSPI. Consequently, with the proposed universal optical setup, both PS-DSPI and SC-DSPI can be used to measure 3D deformation.

\section{Conclusion}

A universal optical setup, with a simple structure, for both PS-DSPI and SC-DSPI is introduced, aiding in the flexibility of full-field 3D deformation measurements. Experimental results show that clear phase maps with regular pattern and reasonable deformation measurement results can be obtained using this setup, verifying the validity of the presented method. Compared to traditional separate PS-DSPI and SC-DSPI setups, the performance of the proposed setup is not degraded. Moreover, its versatility improves the adaptive capacity relative to measuring target variability. Potential DSPI instruments, based on the proposed universal optical setup, will gain more applications and play an important role in practical engineering.

\section{Abbreviations}

3D: Three-dimensional; CMOS: Complementary-metal-oxide-semiconductor; DSPI: Digital speckle pattern interferometry; PS-DSPI: Phase-shifting digital speckle pattern interferometry; PZT: Piezoelectric transducer; SC-DSPI: Spatial-carrier digital speckle pattern interferometry

\section{Acknowledgement}

We express sincere thanks to Mr. Bernard Sia from the Optical Lab of Oakland University, who carefully and thoroughly read the manuscript and provided valuable criticisms.

\section{Funding}

The research is supported by the National Natural Science Foundation of China (Grant No. 51275054), Beijing Municipal Commission of Education (Grant No. KM201511232004), and National Major Scientific Instrument and Equipment Development Project of China (Grant No. 2016YFF0101801).

\section{Authors' contributions}

All authors have participated in the method discussion and result analysis. The experiments are conducted by SW and YF. All authors have read and agreed with the contents of the final manuscript.

\section{Authors' information}

Sijin Wu received his PhD in 2012 and now a faculty member in Beijing Information Science and Technology University. His research interest focuses on optical metrology, such as digital speckle pattern interferometry and digital shearography.

Mingli Dong received her PhD in physical electronics from Beijing Institute of Technology, China. She is a professor and dean in the School of Instrumentation Science and Opto-electronics Engineering at Beijing Information Science and Technology University in China. She has multidisciplinary research experiences including machine vision measurement technology, optical metrology, and biomedical detection technology.

Yao Fang is a master student in Beijing Information Science and Technology University. Her research interest is spatial-carrier digital speckle pattern interferometry.

Lianxiang Yang received his PhD in mechanical engineering from the University of Kassel, Germany, in 1997. He is the director of Optical Laboratory and a professor in the Department of Mechanical Engineering at Oakland University, Rochester, Michigan, USA. He has multidisciplinary research experiences including optical metrology, experimental strain/stress analysis, nondestructive testing, and 3D computer vision. He is a fellow of SPIE, a Changjiang scholar of Hefei University of Technology, and an adjunct professor of Beijing Information Science and Technology University.

\section{Competing interests}

The authors declare that they have no competing interests.

\section{Author details}

${ }^{1}$ School of Instrumentation Science and Opto-electronics Engineering, Beijing Information Science and Technology University, Beijing 100192, China.

${ }^{2}$ Department of Mechanical Engineering, Oakland University, Rochester, MI 48309, USA.

Received: 16 August 2016 Accepted: 13 September 2016 Published online: 20 September 2016

\section{References}

1. Kervran, Y., Sagazan, O.D., Crand, S., et al.: Microcrystalline silicon: Strain gauge and sensor arrays on flexible substrate for the measurement of high deformations. Sensors Actuators A Phys. 236(1), 273-280 (2015)

2. Tiziani, H.J., Pedrini, G.: From speckle pattern photography to digital holographic interferometry. Appl. Opt. 52(1), 30-44 (2013)

3. Gao, Z., Deng, Y., Duan, Y., et al.: Continual in-plane displacement measurement with temporal wavelet transform speckle pattern interferometry. Rev. Sci. Instrum. 83(1), 015107 (2012)

4. Shao, X., Dai, X., He, X.: Noise robustness and parallel computation of the inverse compositional Gauss-Newton algorithm in digital image correlation. Opt. Laser. Eng. 71, 9-19 (2015)

5. Zhu, R., Xie, H., Tang, M., et al.: Reconstruction of planar periodic structures based on Fourier analysis of moiré patterns. Opt. Eng. 54(4), 044102 (2015)

6. Pedrini, G., Osten, W.: Time resolved digital holographic interferometry for investigations of dynamical events in mechanical components and biological tissues. Strain 43, 240-249 (2007)

7. Solís, S.M., Santoyo, F.M., Hernández-Montes, M.S.: 3D displacement measurements of the tympanic membrane with digital holographic interferometry. Opt. Express 20(5), 5613-5621 (2012)

8. Yang, L.X., Xie, X., Zhu, L., et al.: Review of electronic speckle pattern interferometry (ESPI) for three dimensional displacement measurement, Chin. J. Mech. Eng. 27(1), 1-13 (2014)

9. Yang, L.X., Zhang, P., Liu, S., et al.: Measurement of strain distributions in mouse femora with 3D-digital speckle pattern interferometry, Opt. Laser. Eng. 45(8), 843-851 (2007)

10. Bhaduri, B., Kothiyal, M.P., Mohan, N.K.: A comparative study of phase-shifting algorithms in digital speckle pattern interferometry. Optik 119(3), 147-152 (2008)

11. Bhaduri, B., Mohan, N.K., Kothiyal, M.P.: Digital speckle pattern interferometry using spatial phase shifting: influence of intensity and phase gradients, J. Mod. Opt. 55(6), 861-876 (2008) 
12. Zhu, L., Wang, Y., Xu, N., et al.: Real-time monitoring of phase maps of digital shearography. Opt. Eng. 52(10), 101902 (2013)

13. Alvarez, A.S., Ibarra, M.H., Santoyo, F.M., et al.: Strain determination in bone sections with simultaneous 3D digital holographic interferometry. Opt. Laser. Eng. 57, 101-108 (2014)

14. Wang, Y., Sun, J., Li, J., et al.: Synchronous measurement of three-dimensional deformations by multicamera digital speckle patterns interferometry. Opt. Eng. 55(9), 091408 (2016)

15. Kulkarni, R., Rastogi, P.: Multiple phase derivative estimation using autoregressive modeling in holographic interferometry. Meas. Sci. Technol. 26(3), 035202 (2015)

16. Tay, C.J., Quan, C., Chen, W.: Dynamic measurement by digital holographic interferometry based on complex phasor method. Opt. Laser Technol. 41(2), 172-180 (2009)

17. $\mathrm{Wu}, \mathrm{S}, \mathrm{Gao}, \mathrm{X}, \mathrm{LV}, \mathrm{Y}$, et al: Micro deformation measurement using temporal phase-shifting and spatial-carrier digital speckle pattern interferometry, SAE Technical Paper 2016-01-0415, (2016). doi:10.4271/2016-01-0415.

18. Wu, S., Zhu, L., Feng, Q., et al.: Digital shearography with in situ phase shift calibration. Opt. Laser. Eng 50(9), 1260-1266 (2012)

19. Xie, X., Chen, X., Li, J., et al.: Measurement of in-plane strain with dual beam spatial phase-shift digital shearography. Meas. Sci. Technol. 26(11), 115202 (2015)

20. Xie, X., Xu, N., Sun, J., et al.: Simultaneous measurement of deformation and the first derivative with spatial phase-shift digital shearography. Opt. Commun. 286, 277-281 (2013)

21. $\mathrm{Wu}, \mathrm{S}$., Zhu, L., Pan, S., et al.: Spatiotemporal three-dimensional phase unwrapping in digital speckle pattern interferometry. Opt. Lett. 41(5), 1050-1053 (2016)

\section{Submit your manuscript to a SpringerOpen ${ }^{\circ}$ journal and benefit from:}

- Convenient online submission

- Rigorous peer review

- Immediate publication on acceptance

- Open access: articles freely available online

- High visibility within the field

- Retaining the copyright to your article

Submit your next manuscript at $\boldsymbol{s p r i n g e r o p e n . c o m ~}$ 